\title{
Ultrasound-Guided Corticosteroid Injection in Carpal Tunnel Syndrome: Comparison Between Radial and Ulnar Approaches
}

This article was published in the following Dove Press journal: Journal of Pain Research

\author{
Arash Babaei-Ghazani (D) \\ Bijan Forogh' \\ Gholam Reza Raissi \\ Tannaz Ahadi ${ }^{\prime}$ \\ Bina Eftekharsadat ${ }^{2}$ \\ Naseh Yousefi (ID) \\ Shahram Rahimi- \\ Dehgolan (iD) ${ }^{3}$ \\ Katayoun Moradi
}

'Neuromusculoskeletal Research Center, Department of Physical Medicine and Rehabilitation, Iran University of Medical Sciences (IUMS), Tehran, Iran; ${ }^{2}$ Physical Medicine and Rehabilitation Research Center, Department of Physical Medicine and Rehabilitation, Tabriz University of Medical Sciences, Tabriz, Iran; ${ }^{3}$ Physical Medicine and Rehabilitation Department, IKHC Center, Tehran University of Medical Sciences (TUMS), Tehran, Iran
Purpose: To compare two common approaches for ultrasonography (US)-guided injection. Patients and Methods: Sixty patients with mild-to-moderate CTS were included in this double-blind randomized controlled trial (RCT). They received a single shot of corticosteroid injection through either the US-guided in-plane approach: radial or ulnar side. Participants were evaluated using Boston Carpal Tunnel Questionnaire (BCTQ) and visual analogue scale (VAS) for pain, as well as electrodiagnosis (EDX) and US parameters before the intervention, and within 12 weeks of follow-up.

Results: In both groups, all outcomes, except for the electrodiagnostic measures, significantly improved within the follow-up. Pain-VAS and both subscales of BCTQ questionnaire, as our main subjective outcomes, revealed dramatic improvement, with the largest amount of changes in VAS (70\%; comparing to baseline value), and about $37 \%$ for both of BQSS and BQFS scales, all indicating superiority of radial to ulnar in-plane approach. During the first follow-up, we did not detect any remarkable preference between the groups in either subjective or electrodiagnostic variables. However, there was a significant difference at next follow-up time-points in terms of VAS for pain and BQFS favoring radial approach (Table 3). Furthermore, US-measured parameters including nerve-circumference and CSA improved only in the radial in-plane group.

Conclusion: The current data proved that radial in-plane approach for CTS injection could be at least as effective as the more common ulnar in-plane method. Even the pain-relief effect was longer for the radial in-plane approach. Also, patients' functional status and objective variables all revealed better outcomes via the new approach.

Keywords: electromyography, corticosteroid injection, Boston Carpal Tunnel Questionnaire, BCTQ, carpal tunnel syndrome, CTS

\section{Introduction}

Carpal tunnel syndrome (CTS), the most common entrapment neuropathy, is the result of median nerve compression at the distal wrist crease as it passes beneath the transverse carpal ligament. ${ }^{1,2}$ Carpal tunnel also contains nine long flexor tendons beside the mentioned nerve. The bony border of this tunnel is radially formed by the trapezium and ulnarly, by the hook of the hamate. The latter has been always considered as the anatomic landmark of canal outlet; while seeing the pisiform indicates canal inlet in the ultrasonography. We uniformly placed the US-probe immediately distal to pisiform level.

Lifelong prevalence of CTS for general population is about $10 \%$ and $15 \%$ for those with higher occupational risk, resulting in a point prevalence of $2 \%$ among

Katayoun Moradi

Neuromusculoskeletal Research Center,

Department of Physical Medicine and

Rehabilitation, Iran University of Medica

Sciences (IUMS), Tehran, Iran

Email katayoun-moradi69@gmail.com 
the adult population. Women are affected more often than men, nearly three to five folds. ${ }^{3}$ Also, it has been reported as the most common musculoskeletal disorder in most European countries and North America. In 2006, CTS was responsible for $37 \%$ of all the occupational sickness leaves and workers' compensation pay-offs. ${ }^{4}$

Although most cases of CTS are idiopathic, it can occur secondary to conditions such as rheumatoid arthritis (RA), hypothyroidism, trauma especially fracture or dislocation of carpal bones, diabetes mellitus, consumption of oral contraceptive pills, or pregnancy-related conditions. ${ }^{5}$ Patients with CTS often describe symptoms in the distribution of median nerve distal to the wrist which includes burning and tingling sensation, as well as paresthesia in the same area. ${ }^{6}$ As the disease progresses, weakness of thenar muscles can occur resulting in irreversible atrophy in thenar region. ${ }^{1}$

The diagnosis is usually reached on the basis of clinical findings and could be confirmed via the nerve conduction studies (NCS) with sensitivity of about $80-90 \%{ }^{1,7}$ More recently, US has been also employed for the diagnosis and an injection guide. ${ }^{5}$ Different non-surgical treatments can be used for the management of mild-to-moderate CTS. Lifestyle modifications (eg, avoidance of excessive wrist movement and prolonged computer usage) should be considered as the first-line instruction. ${ }^{1}$ The other conservative interventions include night splinting, local and oral corticosteroids, or other medications such as non-steroidal antiinflammatory drugs. ${ }^{8,9}$

One of the most effective methods to alleviate symptoms is corticosteroid injection which has been widely used to achieve a rapid pain relief. ${ }^{10}$ This intervention has been conventionally performed with the guidance of local anatomic landmarks, sometimes called the "blind" technique. However, the risk of nerve injury and damage to other structures such as arteries and veins cannot be completely avoided using this technique. Moreover, physician cannot ensure the accurate placement of the injection needle inside the carpal tunnel using this technique. ${ }^{9}$

US-guided injection can verify the needle position within the carpal tunnel and consequently ensure a successful injection which can lead to a lower risk of median nerve injury. ${ }^{11}$ US provides a real-time image of the nerve, the carpal tunnel, and the surrounding structures. Prior literature have compared US-guided injections against the blind technique and have demonstrated that US-guided ones are associated with higher effectiveness. ${ }^{9}$ Although numerous injection approaches using US- guidance have been described, there is not enough evidence favoring one to others. ${ }^{11}$ Generally, there are two methods of US-probe placement in the wrist: short axis (transverse view) and long-axis (longitudinal view). Although the latter method provides better visualization of the entire median nerve, safety concerns should be considered in the longitudinal method. Transverse scan has been widely used as the injection guidance through different approaches. This study was conducted in order to evaluate the effectiveness and safety of local corticosteroid injection via two US-guided transverse approaches: inplane ulnar and in-plane radial, in terms of symptom relief, functional improvement, as well as changes in electrophysiological and US parameters.

\section{Patients and Methods Study Design and Setting}

This randomized clinical trial was conducted on CTS patients referred to Physical Medicine and Rehabilitation clinic of Rasoul Akram and Firoozgar hospitals between Dec. 2017 and Aug. 2018. Present study complied with all regulations of Helsinki Declaration and was approved by ethics committee of Iran University of Medical Sciences (IUMS) under number IR.IUMS.FMD.REC1396.155559. Also, a written informed consent was obtained from all participants. Our protocol has been fully registered in Iranian registry of clinical trials (IRCT) database under number IRCT20180205038619N1.

\section{Participants}

The history, physical examination and demographic information were taken by a physical medicine and rehabilitation specialist blinded to allocation. Seventy patients with signs and symptoms of CTS including pain and sensory disturbance across the median nerve territory were recruited. Sixty patients with confirmed diagnosis of mildto-moderate CTS, based on electrodiagnostic evaluations, were eligible for the study. In case of bilateral involvement, only the worse symptomatic hand was included for each patient. Our inclusion criteria were as the following: A) age between 15 and 60 years; B) having signs/symptoms of CTS including pain, numbness, paresthesia and/or tingling along the distribution of median nerve in the hand (the first three radial digits and the radial side of the fourth finger). We excluded either of the following cases: severe CTS; thenar muscles atrophy; individuals who had diseases mimicking CTS signs such as polyneuropathy, 
cervical radiculopathy, and thoracic outlet syndrome; a history of prior surgical release or injection for CTS treatment within the last 6 months; allergic reaction to corticosteroid; the presence of skin infection at the site of injection; the willingness of patients to exit from the study at any stage of the project; and not attending any of the follow-up visits.

\section{Randomization and Blinding}

Participants were randomly assigned into two groups using a random number generating software (computer-based). Treatment allocations were concealed using sealed opaque envelopes wrapped by an experienced nurse who was not involved in the recruitment. This led to having two groups with equal number of subjects and balanced distribution of important covariates. All participants, the physician who assessed the outcome measures (K.M.), and the data analyst (S.R.D.) were blinded to the groups.

\section{Interventions}

Participants in both groups were placed in supine position. The skin over the injection site of the affected wrist was prepared with antiseptic. Ultrasound probe was covered with a sterile barrier and was placed transversely (in a shortaxis method) over the distal crease of the affected wrist. Then, a 23-gauge needle containing $1 \mathrm{cc}$ of triamcinolone (40mg per $\mathrm{mL}$ ) was inserted into the carpal tunnel under the ultrasound guidance via either of the two in-plane approaches. For group A, radial approach was applied, surpassing radial artery superficially with puncturing the skin overlaying distal crease and crossing flexor retinaculum to reach the carpal tunnel (Figure 1). However, for group $\mathrm{B}$, injection was performed via ulnar approach, lateral and superficial to ulnar artery, at the level of distal crease (Figure 2). All injections were performed by the same physiatrist (T.A) with 10 years of experience in the field of musculoskeletal injections. After the procedure, the patients were instructed to apply cold pack over the injection site for about 20 mins twice a day. Also, a resting wrist splint was prescribed for all patients for 6 weeks. To ensure the compliance of wearing the splint, a senior resident (K.M.) took the responsibility for reminding patients via periodical phone calls.

\section{Outcome Measures}

Visual analogue scale (VAS) for pain, as the primary outcome measuring tool, and Boston carpal tunnel questionnaire (BCTQ) were used for evaluating subjective efficacy

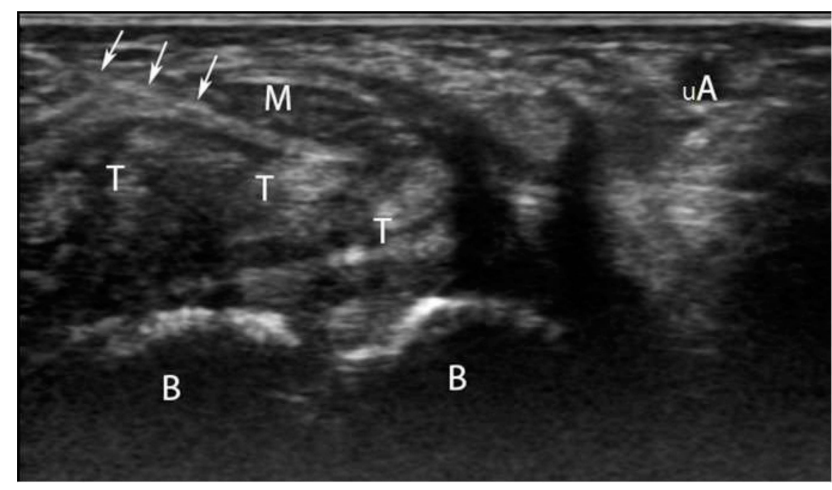

Figure I Ultrasound-guided CTS injection from radial side in transverse (short axis) view ( $M$, median nerve; $T$, tendons; $B$, carpal bones; $u A$, ulnar artery; white arrows, needle).

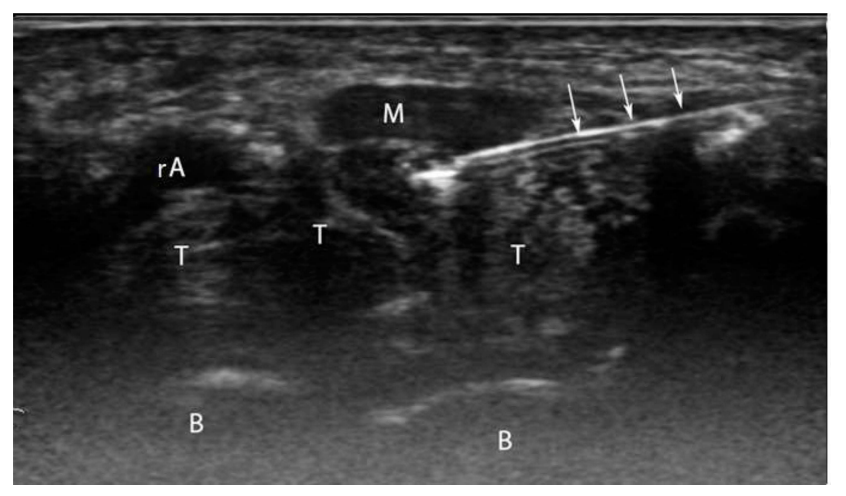

Figure 2 Ultrasound-guided injection inside the carpal tunnel from ulnar side in transverse (short axis) view (M, median nerve; T, tendons; B, carpal bones; $r A$, radial artery; white arrows, needle).

of the two approaches, while the electrodiagnostic and sonographic parameters were utilized for determining the objective improvement. These measures were assessed from all patients before the injection and at follow-up visits after 2, 6 and 12 weeks.

Pain intensity was measured using a 10-cm scale (painVAS) in which " 0 " indicates no pain and " 10 " represents the worst pain ever experienced. Patients were asked to mark a place on the VAS that corresponded to their average pain level at resting position during the last 2 days.

BCTQ is a patient-based outcome measure that has been developed specifically for patients with CTS. It has two distinct scales: the Boston Questionnaire Symptom Severity Scale (BQ-SS) which has 11 items and the Boston Questionnaire Functional Status Scale (BQ-FS) containing 8 items rated for the degree of difficulty, both subscales on a 5-point Likert scale. Each scale generates a final score (sum of individual scores divided by the number of items) which ranges from 1 to 5 , with a higher score indicating greater disability. The BCTQ has been employed as an 
outcome measure in clinical studies and has already undergone extensive testing for validity and reliability, both in original version $^{12}$ and in Persian translation. ${ }^{13}$

The electrodiagnostic evaluation was performed with an electromyography machine (Natus Synergy ${ }^{\circledR}$ Ultrapro S100). We measured distal latency and amplitude of both the compound muscle action potential (CMAP) and sensory nerve action potential (SNAP) for the median nerve. The measurements were performed based on the standards provided by Dumitru and Amato. ${ }^{14}$ Ulnar and radial nerve conduction study and the electromyography of the required muscles was also conducted to eliminate other deceptive pathologies such as peripheral polyneuropathies, cervical radiculopathy or plexopathies.

The ultrasound examination was performed with a Hitachi ARIETTA V60 ultrasound machine (Hitachi ${ }^{\circledR}$ Aloka Medical Systems, Tokyo, Japan). The patients were positioned in the upright sitting with their hands on a pillow, the forearm supinated and the wrist in slight dorsiflexion using a rolled-up towel. A high-frequency linear transducer (7-12 MHz) was then placed transversely along the distal crease of the wrist. The median nerve was identified just after seeing the pisiform, as the inlet of tunnel, and its cross-sectional area (CSA) and circumference were determined. The median nerve CSA was measured by tracing the internal border of perineural echogenic rim over the median nerve. For each person, three measurements were taken and the mean value was considered for analysis. Cross-sectional area $\geq 10 \mathrm{~mm}^{2}$ at the mentioned level was the most common parameter to diagnose CTS on US, and its sensitivity has been reported to be as high as $97.9 \%$ for CTS diagnosis. ${ }^{5}$

\section{Statistical Analysis}

Statistical analysis was performed in SPSS software version 22.0 (SPSS Inc. ${ }^{\circledR}$, Chicago, IL, USA). Kolmogorov-Smirnov test showed normal data distribution for all variables and the Student's $t$-test was used to compare the parametric comparison of data at baseline. Cross tabs and Chi-square test were used to explore and compare the possible differences between the two groups regarding sex and the involved side. The significant level was set as $\mathrm{P}<0.05$.

\section{Results}

Seventy patients with consistent signs and symptoms of CTS were assessed for eligibility. Among them, 10 subjects who did not meet inclusion criteria or declined to participate in the study were excluded. The remaining 60 patients were randomly divided into two equal groups (Figure 3). There existed no significant differences in the demographic and baseline clinical characteristics of the patients between the two groups (Table 1). The mean age of participants was $52.67 \pm 8.6$ years in ulnar group and $51.70 \pm 10.5$ years in the radial group. The mean BMI values in ulnar and radial groups were $29.31 \pm 3.3$ and $30.16 \pm 3.8 \mathrm{~kg} / \mathrm{m}^{2}$, respectively. At the beginning of the study, there was no significant difference between the two groups of patients regarding pain-VAS and BCTQ, as well as the electrodiagnostic and sonographic parameters (Table 1).

Table 2 has demonstrated the effectiveness of corticosteroid injection within each study group at different time-points. VAS scores showed a significant and equal pain-relief effect in both study groups within 2 and 6 weeks follow-up, compared to baseline. But at the last visit, interestingly, patients treated with ulnar inplane approach experienced a significant worsening of pain after the 6th and 12th weeks of follow up $(2.93 \pm 2.3$ and $4.20 \pm 1.4$, respectively), after the initial improvement in their pain (from $7.60 \pm 1.6$ to $2.83 \pm 2.3$ ) during the first 2 weeks (Table 3). In contrast, patients in the radial group, reported a persistent pain relief until the 6th week of follow-up (from $7.33 \pm 1.7$ in baseline to 2.13 \pm 1.5 ); and eventually this improvement reached to a plateau in the last visit $(2.43 \pm 1.7)$. This was associated with a statistically significant superiority of radial approach at the last visit (Table 3 ).

A similar trend was observed for BQFS. Improvement in both groups was evidently observed at all time-points; without any preference between the groups during the first 2 weeks. But at the 6th wand 12th weeks of follow up, the difference in BQFS scores became significant and the radial in-plane approach surpassed the ulnar group (Table 3). The other subscale of Boston questionnaire, ie, BQSS, did not reveal any obvious superiority between the two approaches (Table 3), although the improvement was remarkable within all sessions for both groups (Table 2).

Among the electrodiagnostic parameters, CMA and SNAP latency as well as CMAP amplitude were not associated with a detectable improvement at all. SNAP latency showed a subtle preference in favor of ulnar in-plane approach with about $10.0 \%$ improvement (Table 2). However, none of these three parameters achieved a clinically important level of changes. While the other one, ie, SNAP amplitude revealed a statistically significant 


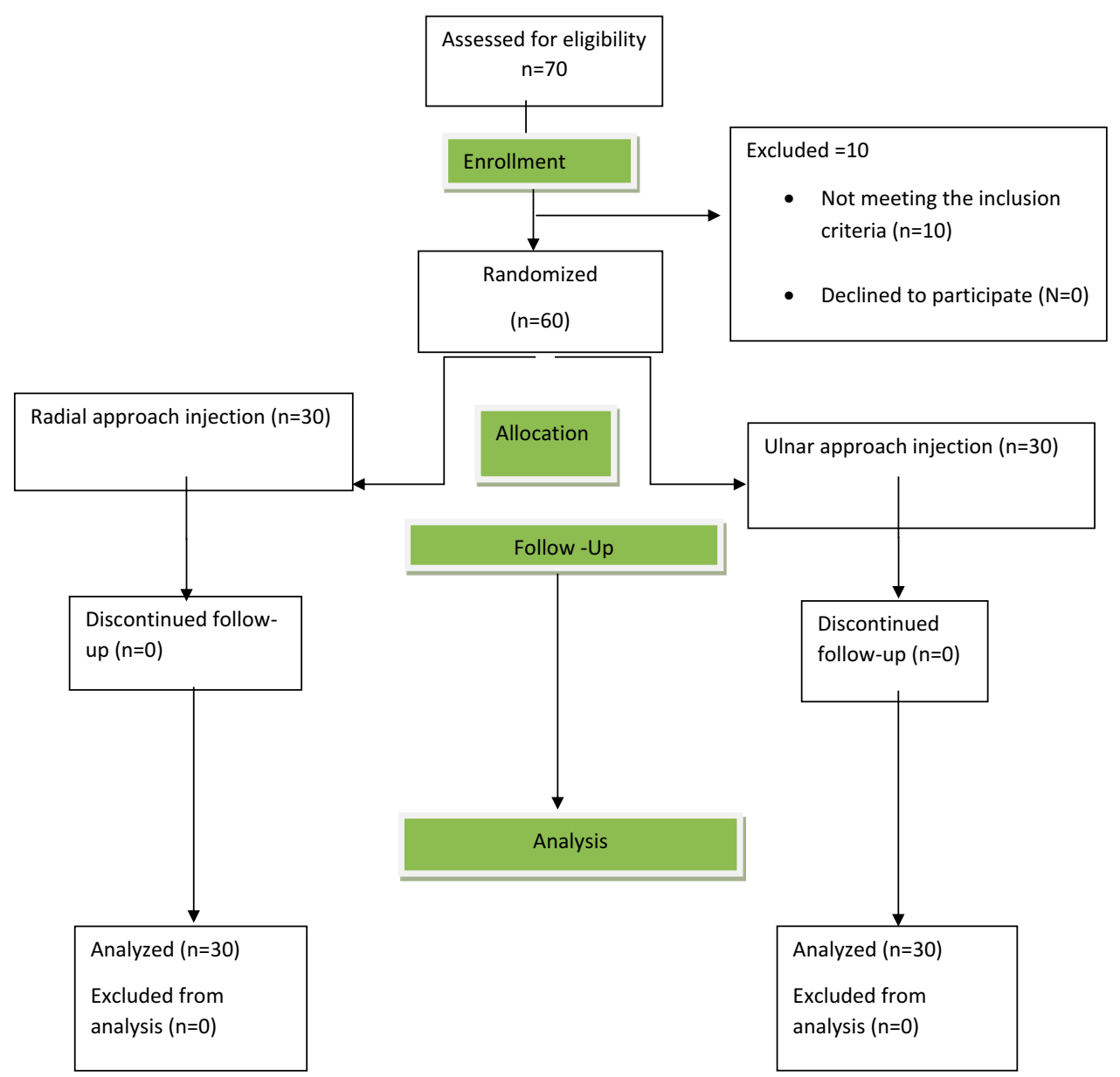

Figure 3 Flowchart of included and excluded participants.

change. It was evidently better in the radial group (14.0\% to $28.7 \%$ improvement) compared to the ulnar (8.3\% to $13.5 \%)$ approach (Table 2). Moreover, both of the two sonographic variables showed a significant superiority favoring radial inplane approach (with the mean percent of changes calculated about $19 \%$ for nerve-CSA and circumference of median). Besides, there was not any significant improvement within the ulnar in-plane group for these two US-parameters.

From the temporal point of view, as we could find out in Table 3, except for nerve-circumference and CSA, there existed no significant difference between the two groups after 2-weeks follow-up. The mentioned US-parameters showed a small preference in favor of the radial in-plane approach, and this superiority remained significant until the 6th week, while BQFS revealed another significant difference (Table 3). On the other hand, BQSS, as well as both the CMAP latency and amplitude did not discover any significant difference between the two groups even after 12 weeks of follow up. However, as mentioned earlier, pain-VAS score, BQFS, and SNAP amplitude were significantly better in radial approach compared to the ulnar group. Exceptionally, a significant superiority was noted in SNAP latency for ulnar approach (Table 3). Albeit it should be kept in mind that SNAP latency improvement was not significant within the two groups at all.

To summarize, the radial in-plane approach was associated with better effectiveness regarding all outcome measures, except for one variable (Table 2). Among them, BQSS, as well as both CMAP amplitude and latency values had nonsignificant $\mathrm{P}$ values (Table 3). However, the radial in-plane approach was evidently superior to the ulnar in terms of VAS for pain, BQFS, SNAP amplitude, nerve-circumference and CSA. The most important difference was calculated for painVAS that showed about $67 \%$ improvement in the radial group, versus the ulnar group with $45 \%$ changes comparing to baseline (Table 2). On the other hand, the only significant difference in favor of the ulnar approach was achieved for SNAP latency that was slightly lower in the ulnar group (Table 3). Furthermore, US-measured parameters including 
Table I Baseline Characteristics of All Participants

\begin{tabular}{|c|c|c|c|}
\hline Characteristics & $\begin{array}{l}\text { Ulnar } \\
\text { Approach }\end{array}$ & $\begin{array}{l}\text { Radial } \\
\text { Approach }\end{array}$ & P-value \\
\hline Number of participants & 30 & 30 & \\
\hline Age $_{[y]}$ Mean (SD) & $52.67(8.64)$ & $51.70(10.58)$ & 0.700 \\
\hline $\mathrm{BMI}_{\left[\mathrm{kg} / \mathrm{m}^{2}\right]}$ Mean (SD) & $29.31(3.36)$ & $30.16(3.80)$ & 0.360 \\
\hline VAS for pain Mean (SD) & $7.60(1.61)$ & $7.33(1.73)$ & 0.539 \\
\hline BQSS Mean (SD) & $2.38(0.62)$ & $2.52(0.76)$ & 0.441 \\
\hline BQFS Mean (SD) & $2.45(0.54)$ & $2.52(0.76)$ & 0.200 \\
\hline SNAP latency [ms] Mean (SD) & $4.49(0.53)$ & $4.59(0.61)$ & 0.513 \\
\hline SNAP amplitude ${ }_{[\mu \mathrm{v}]}$ Mean (SD) & $18.12(6.04)$ & $16.89(5.21)$ & 0.403 \\
\hline CMAP latency $[\mathrm{ms}]$ Mean $(\mathrm{SD})$ & $4.65(0.70)$ & $4.7 I(0.79)$ & 0.755 \\
\hline CMAP amplitude ${ }_{[\mathrm{mv}]}$ Mean (SD) & $8.55(2.08)$ & $7.56(2.7 \mathrm{I})$ & 0.121 \\
\hline Nerve circumference ${ }_{[\mathrm{cm}]}$ Mean (SD) & $0.15(0.02)$ & $0.14(0.04)$ & 0.155 \\
\hline Nerve CSA $\left[\mathrm{mm}^{2}\right]$ Mean (SD) & $17.08(2.37)$ & $16.61(3.62)$ & 0.555 \\
\hline
\end{tabular}

Abbreviations: BMI, body mass index; VAS, visual analogue scale; BQSS, Boston Questionnaire Symptom Severity Scale; BQFS, Boston Questionnaire Functional Status Scale; SNAP, sensory nerve action potential; CMAP, compound muscle action potential; CSA, cross-sectional area; SD, standard deviation; $y$, year; kg, kilogram; $\mathrm{m}^{2}$, meter square; $\mathrm{cm}$, centimeter; $\mathrm{mm}^{2}$, millimeter square; $\mu \mathrm{v}$, microvolt; $\mathrm{mv}$, millivolt; $\mathrm{ms}$, millisecond.

nerve-circumference and CSA improved only in the radial in-plane group, while the ulnar approach was not associated with a remarkable decline. Lastly, it should be noted that all patients tolerated the injection procedure well and no serious adverse event was observed in the current study.

\section{Discussion}

Corticosteroid injection is a well-studied and effective intervention for patients with mild and moderate CTS. It is a straightforward procedure with minimum side effects that can result in remarkable improvement of symptoms and functional status even within the first week after injection. ${ }^{5}$ In a routine practice, this intervention is often performed blindly, with a high risk of damaging the nerve and vulnerable structures such as tendons and vessels. ${ }^{15}$ Racasan and Dubert ${ }^{16}$ reported that the median nerve is at risk of damage if the injection is performed within $1 \mathrm{~cm}$ on either the ulnar or the radial side of the palmaris longus tendon with a blind procedure. The Ultrasound guidance might improve the accuracy and the effectiveness of steroid injection in CTS. ${ }^{10}$ In the US-guided injection, the structure and location can be visualized by physicians, so that they can reach the carpal tunnel without any damage to vulnerable tissues, and the distribution of the injected substance can be seen to ensure the perfect location of injection. ${ }^{11}$

Many approaches to carpal tunnel injection have been studied by several authors. Formerly, one of the most widely used techniques was the midline longitudinal US- guided injection. This method had some disadvantages; especially, when we aim to perform perineural injection, or as sometimes called hydro-dissection. Previous research advocated that a short-axis or transverse scan is superior to a long-axis scan considering that the US image in longitudinal scan might confuse operators between swollen nerve fascicles, muscles, and inflamed tendons in the same plane; hence, raising concerns regarding the incidence of nerve trauma due to injections in the long-axis (longitudinal) scan.

Smith et al developed the in-plane ulnar approach for the administration of US-guided carpal tunnel injection. ${ }^{17}$ Several studies showed that US-guided local steroid injection using an in-plane ulnar approach in the CTS might be more effective than out-of-plane injections. ${ }^{11,18,19}$ Nonetheless, other injection approaches had not been evaluated in the prior studies. The primary purpose of this study was to compare the efficacy and safety of in-plane radial versus the more common ulnar in-plane approach in US-guided carpal tunnel injection.

Our study proved that in both treatment groups, all outcomes, except for almost all of electrodiagnostic measures, significantly improved within 2, 6 and 12 weeks of follow-up (Table 2). Pain-VAS and both subscales of BCTQ questionnaire, as our main subjective outcomes, revealed dramatic improvement, with the largest amount of changes in VAS (70\%; comparing to baseline value), and about $37 \%$ for both of BQSS and BQFS scales, all indicating superiority of radial to ulnar in-plane approach (Table 2). During the first followup, we did not detect any remarkable preference between the groups in either subjective or electrodiagnostic measuring tools. However, there was a significant difference at the second follow-up in terms of VAS for pain and BQFS; and at the third follow-up in terms of BQFS, all favoring radial approach (Table 3).

The first RCT evaluating ulnar in-plane approach was performed by Lee et al. ${ }^{11}$ According to that study, outcome measuring tools including BCTQ, median nerve NCS parameters, and flattening ratio and CSA of median nerve revealed a significant improvement at week 4 of the followup for the in-plane ulnar approach group compared with the out-of-plane ulnar approach. It was a lasting improvement even after 12 weeks which was consistent with our findings. We proved that patients' symptoms evaluated by BCQT (BQSS and BQFS), VAS for pain, and sonographic and nerve conduction parameters were relieved after 2 weeks which lasted until the 12th week of the treatment. 
Table 2 Within-Groups Comparison of Outcome Measures

\begin{tabular}{|c|c|c|c|}
\hline Variable/Comparison & Baseline to 2 Weeks & Baseline to 6 Weeks & Baseline to 12 Weeks \\
\hline \multicolumn{4}{|c|}{ Improvement in pain-VAS (MD) } \\
\hline Radial & $4.12 * *(56.2 \%)$ & $5.20 * *(70.9 \%)$ & $4.90 * *(66.8 \%)$ \\
\hline Ulnar & $4.77 * *(62.7 \%)$ & $4.67 * *(61.4 \%)$ & $3.40 * *(44.7 \%)$ \\
\hline \multicolumn{4}{|c|}{ Improvement in BQSS $(\mathrm{MD})$} \\
\hline Radial & $0.90 * *(35.7 \%)$ & $0.95 * *(37.6 \%)$ & $0.90 * *(35.7 \%)$ \\
\hline Ulnar & $0.77 * *(32.3 \%)$ & $0.79 * *(33.1 \%)$ & $0.53 * *(22.2 \%)$ \\
\hline \multicolumn{4}{|c|}{ Improvement in BQFS (MD) } \\
\hline Radial & $0.55^{* *}(24.8 \%)$ & $0.80 * *(36.1 \%)$ & $0.72 * *(32.5 \%)$ \\
\hline Ulnar & $0.64 * *(26.1 \%)$ & $0.56 * *(22.8 \%)$ & $0.48 * *(19.5 \%)$ \\
\hline \multicolumn{4}{|c|}{ Improvement in SNAP latency [ms] (MD) } \\
\hline Radial & $0.23(5.0 \%)$ & $0.25(5.4 \%)$ & $0.20(4.3 \%)$ \\
\hline Ulnar & $0.21(4.6 \%)$ & $0.31(6.9 \%)$ & $0.45(10.0 \%)$ \\
\hline \multicolumn{4}{|c|}{ Improvement in SNAP amplitude $[\mu \mathrm{v}]$ (MD) } \\
\hline Radial & $1.54(9.1 \%)$ & $2.38 * *(14.0 \%)$ & $4.85 * *(28.7 \%)$ \\
\hline Ulnar & $2.14(11.8 \%)$ & $2.45(13.5 \%)$ & $1.5 \mathrm{I}(8.3 \%)$ \\
\hline \multicolumn{4}{|c|}{ Improvement in CMAP latency [ms] (MD) } \\
\hline Radial & $0.34(7.1 \%)$ & $0.22(4.6 \%)$ & $0.38(8.0 \%)$ \\
\hline Ulnar & $0.45(9.7 \%)$ & $0.49(10.5 \%)$ & $0.21(4.5 \%)$ \\
\hline \multicolumn{4}{|c|}{ Improvement in CMAP amplitude [mv] (MD) } \\
\hline Radial & $0.25(3.3 \%)$ & $0.15(1.9 \%)$ & $0.40(5.2 \%)$ \\
\hline Ulnar & $0.07(0.8 \%)$ & $0.29(3.3 \%)$ & $0.15(1.7 \%)$ \\
\hline \multicolumn{4}{|c|}{ Improvement in nerve circumference ${ }_{[\mathrm{cm}]}$ (MD) } \\
\hline Radial & $0.020 * *(14.2 \%)$ & $0.027 * *(19.2 \%)$ & $0.006(4.2 \%)$ \\
\hline Ulnar & $0.008(5.3 \%)$ & $0.007(4.6 \%)$ & $0.002(1.3 \%)$ \\
\hline \multicolumn{4}{|c|}{ Improvement in nerve CSA $\left[\mathrm{mm}^{2}\right]$ (MD) } \\
\hline Adial & $1.92 * *(11.5 \%)$ & $2.38 * *(14.3 \%)$ & $3.12 * *(18.7 \%)$ \\
\hline Ulnar & $1.20(7.0 \%)$ & $1.05(6.1 \%)$ & $1.33(7.7 \%)$ \\
\hline
\end{tabular}

Notes: **P values $\leq 0.05$ using "Repeated Measures" test (representing a significant improvement within the radial and ulnar groups). The best percent of each variable has been indicated in bold format.

Abbreviations: MD, mean difference; VAS, visual analogue scale; BQSS, Boston Questionnaire Symptom Severity Scale; BQFS, Boston Questionnaire Functional Status Scale; SNAP, sensory nerve action potential; CMAP, compound muscle action potential; CSA, cross-sectional area; SD, standard deviation; cm, centimeter; mm², millimeter square; $\mu \mathrm{v}$, microvolt; mv, millivolt; ms, millisecond.

The US-guided ulnar approach has been adopted by several authors the majority of whom reported a significant enhancement in pain-VAS, BCTQ and electrophysiological parameters compared to landmark-guided approaches. ${ }^{20-22}$ Üstün et $\mathrm{al}^{20}$ used the out-of-plane ulnar technique, whereas Makhlouf et $\mathrm{al}^{21}$ and Eslamian et $\mathrm{al}^{22}$ conducted their studies with in-plane ulnar corticosteroid injection. Üstün et $\mathrm{al}^{20}$ and Makhlouf et $\mathrm{al}^{21}$ suggested that US-guided steroid injection may be more effective than blind injections in contrast to Eslamian et $\mathrm{al}^{22}$ who reported that there was not a significant difference between US-guided and landmark-guided (blind) injections for CTS. They stated that the expertise of the specialist conducting blind injections could be a decisive factor in the efficiency of the blind CTS injection, which requires further studies.

In a meta-analysis conducted by Chen et $\mathrm{al}^{18}$ comparing 4 different injection techniques (proximal and distal blind approaches versus in-plane and out-of-plane ulnar methods), authors finally concluded that the US-guided in-plane ulnar approach for corticosteroid injection was the best one in order to achieve a good clinical response (in terms of amount of changes in BCTQ) at short-term follow-up period. The present authors in another meta-analysis, ${ }^{23}$ compared USguided and landmark-guided local corticosteroid injection for CTS. Through pooled data, they demonstrated that USguided injection was more effective in BQSS improvement, 
Table 3 The Between-Groups Analysis of Outcome Measures at Different Time-Points

\begin{tabular}{|c|c|c|c|c|}
\hline Variable & Baseline & After 2 Weeks & After 6 Weeks & After 12 Weeks \\
\hline \multicolumn{5}{|l|}{ VAS for pain mean (SD) } \\
\hline Radial & $7.33(1.73)$ & $3.21(2.22)$ & $2.13(1.55)$ & $2.43(1.70)$ \\
\hline Ulnar & $7.60(1.61)$ & $2.83(2.31)$ & $2.93(2.36)$ & $4.20(1.47)$ \\
\hline \multicolumn{5}{|l|}{ BQSS $_{\text {mean (SD) }}$} \\
\hline Radial & $2.52(0.76)$ & $1.62(0.65)$ & $1.56(0.66)$ & $1.62(0.47)$ \\
\hline Ulnar & $2.38(0.62)$ & $1.61(0.45)$ & $1.59(0.43)$ & $1.84(0.39)$ \\
\hline \multicolumn{5}{|l|}{ BQFS mean (SD) } \\
\hline Radial & $2.21(0.85)$ & $1.66(0.70)$ & $1.41(0.45)$ & $1.49(0.44)$ \\
\hline Ulnar & $2.45(0.54)$ & $1.81(0.75)$ & $1.89(0.67)$ & $1.97(0.63)$ \\
\hline \multicolumn{5}{|l|}{ SNAP latency [ms] mean (SD) } \\
\hline Radial & $4.59(0.61)$ & $4.36(0.60)$ & $4.34(0.61)$ & $4.39(0.35)$ \\
\hline Ulnar & $4.49(0.53)$ & $4.28(0.67)$ & $4.19(0.70)$ & $4.05(0.67)$ \\
\hline \multicolumn{5}{|l|}{ SNAP Amplitude ${ }_{[\mu \mathrm{v}]}$ mean (SD) } \\
\hline Radial & $16.89(5.21)$ & $18.43(5.06)$ & $20.81(4.02)$ & 21.74 (3.69) \\
\hline Ulnar & $18.12(6.04)$ & $20.26(8.79)$ & $20.57(7.44)$ & $19.63(3.66)$ \\
\hline \multicolumn{5}{|l|}{ CMAP latency [ms]mean (SD) } \\
\hline Radial & $4.73(0.77)$ & $4.39(0.75)$ & $4.51(1.96)$ & $4.35(0.66)$ \\
\hline Ulnar & $4.63(0.62)$ & $4.18(0.22)$ & $4.15(0.23)$ & $4.42(0.58)$ \\
\hline \multicolumn{5}{|l|}{ CMAP Amplitude [mv]mean (SD) } \\
\hline Radial & $7.56(2.7 I)$ & $7.81(2.44)$ & $7.71(2.18)$ & $7.97(2.13)$ \\
\hline Ulnar & $8.55(2.10)$ & $8.48(2.39)$ & $8.27(2.40)$ & $8.40(1.70)$ \\
\hline \multicolumn{5}{|l|}{ Nerve circumference $[\mathrm{cm}]$ mean (SD) } \\
\hline Radial & $0.14(0.04)$ & $0.12(0.03)$ & $0.11(0.03)$ & $0.13(0.11)$ \\
\hline Ulnar & $0.15(9.02)$ & $0.14(0.02)$ & $0.14(0.02)$ & $0.15(0.03)$ \\
\hline \multicolumn{5}{|l|}{ Nerve CSA $\left[\mathrm{mm}^{2}\right]$ mean (SD) } \\
\hline Radial & $16.61(3.62)$ & $14.69(2.17)$ & $14.23(2.16)$ & $13.49(2.09)$ \\
\hline Ulnar & $17.08(2.37)$ & $15.88(2.21)$ & $16.03(2.10)$ & $15.75(1.66)$ \\
\hline
\end{tabular}

Notes: Bold format: $\mathrm{P}$ values $\leq 0.05$ using "The Student's $t$-test" (representing a significance difference between radial and ulnar approaches).

Abbreviations: VAS, visual analogue scale; BQSS, Boston Questionnaire Symptom Severity Scale; BQFS, Boston Questionnaire Functional Status Scale; SNAP, sensory nerve action potential; CMAP, compound muscle action potential; CSA, cross-sectional area; SD, standard deviation; cm, centimeter; mm², millimeter square; $\mu \mathrm{v}$, microvolt; mv, millivolt; ms, millisecond.

whereas no significant difference was observed between the 2 methods in terms of the amplitude and latency of BQFS, CMAP and SNAP. Also, Omar et $\mathrm{al}^{24}$ compared the clinical outcomes of the US-guided injection versus blind injections in 30 patients with CTS. Patients with US-guided injection had significant improvement of clinical (BQSS and BQFS), neurophysiological, and ultrasound parameters (nerve-CSA and flattening ratio) than those with blind injections. While, in our study, three out of four electrodiagnostic measures did not reveal a significant improvement within the two USguided approaches.

Some studies evaluate different US-guided techniques. Babaei-Ghazani et al $^{25}$ compared in-plane ulnar US-guided corticosteroid injection with "above" and "below" the median nerve approaches and concluded that both techniques were effective in symptom relief and functional improvement; as well they observed almost equal change in electrodiagnostic and sonographic parameters. In our study, we used in-plane radial versus the widely used ulnar approach for the carpal tunnel injection. It is the first report evaluating radial technique with the use of objective measurement (ie, electrophysiological and ultrasonographic parameters), therefore we found no similar study to compare findings against. According to our results, both approaches were successful in improving the subjective parameters (pain-VAS and BCTQ) and US-measured variables (nerve-circumference and CSA). While almost all electrodiagnostic measures were equal between the two groups, the radial approach showed a significant superiority in the improvement of other mentioned measures. 
The current study had some limitations. Primarily, we were concerned about the small sample size and the lack of longterm follow-up beyond 12 weeks. Therefore, we recommend additional studies with larger sample size and longer followups. Future investigations could also include other sonographic parameters such as median nerve vascularity, mobility, and echogenicity, as well as flattening ratio and flexor retinaculum thickness.

\section{Conclusions}

The current data proved that radial in-plane approach for CTS injection could be at least as effective as the more common ulnar in-plane method. Even the pain-relief effect was longer for the radial in-plane approach. Also, patients functional status and objective variables all revealed better outcomes via the new approach.

\section{Data Sharing Statement}

The authors are ready to share the de-identified file of data in excel format and all other study-related documents. It would be available on the demand of editorial board via the corresponding authors' email.

\section{Disclosure}

This study had no funding source and authors declare that they had no conflict of interests. This article has been extracted from the thesis written by Dr. Katayoun Moradi in School of Medicine, Iran University of Medical Sciences (IUMS).

\section{References}

1. Padua L, Coraci D, Erra C, et al. Carpal tunnel syndrome: clinical features, diagnosis, and management. Lancet Neurol. 2016;15 (12):1273-1284. doi:10.1016/S1474-4422(16)30231-9

2. Wang JC, Liao KK, Lin KP, et al. Efficacy of combined ultrasound-guided steroid injection and splinting in patients with carpal tunnel syndrome: a randomized controlled trial. Arch Phys Med Rehabil. 2017;98(5):947-956. doi:10.1016/j.apmr.2017.01.018

3. Klauser AS, Buzzegoli T, Taljanovic MS, et al. Nerve entrapment syndromes at the wrist and elbow by sonography. Semin Musculoskelet Radiol. 2018;22(3):344-353. doi:10.1055/s-0038-1641577

4. Petrover D, Richette P. Treatment of carpal tunnel syndrome: from ultrasonography to ultrasound guided carpal tunnel release. Joint Bone Spine. 2018;85(5):545-552. doi:10.1016/j.jbspin.2017.11.003

5. McDonagh C, Alexander M, Kane D. The role of ultrasound in the diagnosis and management of carpal tunnel syndrome: a new paradigm. Rheumatology (Oxford). 2015;54(1):9-19. doi:10.1093/rheumatology/keu275

6. Chen PC, Wang LY, Pong YP, Hsin YJ, Liaw MY, Chiang CW. Effectiveness of ultrasound-guided vs direct approach corticosteroid injections for carpal tunnel syndrome: a double-blind randomized controlled trial. J Rehabil Med. 2018;50(2):200-208. doi:10.2340/16501977-2308

7. Borire AA, Hughes AR, Lueck CJ, Colebatch JG, Krishnan AV. Sonographic differences in carpal tunnel syndrome with normal and abnormal nerve conduction studies. J Clin Neurosci. 2016;34:77-80. doi:10.1016/j.jocn.2016.05.024
8. Carlson H, Colbert A, Frydl J, Arnall E, Elliot M, Carlson N. Current options for nonsurgical management of carpal tunnel syndrome. Int J Clin Rheumtol. 2010;5(1):129-142. doi:10.2217/IJR.09.63

9. Guo XY, Xiong MX, Lu M, et al. Ultrasound-guided needle release of the transverse carpal ligament with and without corticosteroid injection for the treatment of carpal tunnel syndrome. J Orthop Surg Res. 2018;13(1):69. doi:10.1186/s13018-018-0771-8

10. Evers S, Bryan AJ, Sanders TL, Selles RW, Gelfman R, Amadio PC. Effectiveness of ultrasound-guided compared to blind steroid injections in the treatment of carpal tunnel syndrome. Arthritis Care Res (Hoboken). 2017;69(7):1060-1065. doi:10.1002/acr.23108

11. Lee JY, Park Y, Park KD, Lee JK, Lim OK. Effectiveness of ultrasound-guided carpal tunnel injection using in-plane ulnar approach: a prospective, randomized, single-blinded study. Medicine (Baltimore). 2014;93(29):e350. doi:10.1097/MD.0000000000000350

12. Leite JC, Jerosch-Herold C, Song F. A systematic review of the psychometric properties of the Boston Carpal tunnel questionnaire. BMC Musculoskelet Disord. 2006;7:78. doi:10.1186/1471-2474-7-78

13. Rezazadeh A, Bakhtiary AH, Samaei A, Moghimi J. Validity and reliability of the Persian Boston questionnaire in Iranian patients with carpal tunnel syndrome. Koomesh. 2014;15(2):138-145.

14. Dumitru D, Amato AA, Zwarts M, editors. Electrodiagnostic Medicine. 2nd ed. Philadelphia: Hanley \& Belfus; 2001.

15. Dabees N, El-Saadany H, El-Barbary A, Ammar AS. Ultrasound guided steroids injection for carpal tunnel syndrome. Int $J$ Med Imaging. 2015;3(4):75-81. doi:10.11648/j.ijmi.20150304.12

16. Racasan $\mathrm{O}$, Dubert $\mathrm{T}$. The safest location for steroid injection in the treatment of carpal tunnel syndrome. J Hand Surg Br. 2005;30 (4):412-414. doi:10.1016/j.jhsb.2005.04.009

17. Smith J, Wisniewski SJ, Finnoff JT, Payne JM. Sonographically guided carpal tunnel injections: the ulnar approach. $J$ Ultrasound Med. 2008;27(10):1485-1490. doi:10.7863/jum.2008.27.10.1485

18. Chen PC, Chuang $\mathrm{CH}$, Tu YK, Bai CH, Chen CF, Liaw M A Bayesian network meta-analysis: comparing the clinical effectiveness of local corticosteroid injections using different treatment strategies for carpal tunnel syndrome. BMC Musculoskelet Disord. 2015;16(1):394. doi:10.1186/s12891-015-0815-8

19. Kim DH, Jang JE, Park BK. Anatomical basis of ulnar approach in carpal tunnel injection. Pain Physician. 2013;16(3):E191-E198.

20. Ustün N, Tok F, Yagz AE, et al. Ultrasound-guided vs. blind steroid injections in carpal tunnel syndrome: a single-blind randomized prospective study. Am J Phys Med Rehabil. 2013;92(11):999-1004. doi:10.1097/PHM.0b013e31829b4d72

21. Makhlouf T, Emil NS, Sibbitt WL Jr, Fields RA, Bankhurst AD. Outcomes and cost-effectiveness of carpal tunnel injections using sonographic needle guidance. Clin Rheumatol. 2014;33(6):849-858. doi:10.1007/s10067-013-2438-5

22. Eslamian F, Eftekharsadat B, Babaei-Ghazani A, Jahanjoo F, Zeinali M. A randomized prospective comparison of ultrasound-guided and landmark-guided steroid injections for Carpal Tunnel syndrome. J Clin Neurophysiol. 2017;34(2):107-113. doi:10.1097/WNP.0000000000000 342

23. Babaei-Ghazani A, Roomizadeh P, Forogh B, et al. Ultrasound-guided versus landmark-guided local corticosteroid injection for carpal tunnel syndrome: a systematic review and meta-analysis of randomized controlled trials. Arch Phys Med Rehabil. 2018;99(4):766-775. doi:10.1016/j.apmr.2017.08.484

24. Omar G, Ali F, Ragaee A, Darwiesh A. Ultrasound-guided injection of carpal tunnel syndrome: a comparative study to blind injection. Egypt Rheumatol. 2018;40(2):131-135. doi:10.1016/j.ejr.2017.09.001

25. Babaei-Ghazani A, Nikbakht N, Forogh B, et al. Comparison between effectiveness of ultrasound-guided corticosteroid injection above versus below the median nerve in mild to moderate carpal tunnel syndrome: a randomized controlled trial. Am J Phys Med Rehabil. 2018;97(6):407-413. doi:10.1097/PHM.0000000000000877 


\section{Publish your work in this journal}

The Journal of Pain Research is an international, peer reviewed, open access, online journal that welcomes laboratory and clinical findings in the fields of pain research and the prevention and management of pain. Original research, reviews, symposium reports, hypothesis formation and commentaries are all considered for publication. The manuscript management system is completely online and includes a very quick and fair peer-review system, which is all easy to use. Visit http:// www.dovepress.com/testimonials.php to read real quotes from published authors.

Submit your manuscript here: https://www.dovepress.com/journal-of-pain-research-journal 